\title{
Measurement of Transcutaneous Bilirubin with Bilicheck as a Jaundice Screening Method in Neonates in Pediatric Emergency Departments
}

\author{
Concepción Míguez*, Mercedes Fariñas Salto, Rafael Marañón \\ Pediatric Emergency Department, Hospital General Universitario Gregorio Marañón, Madrid, Spain \\ Email: ${ }^{\text {c.miguez09@gmail.com }}$
}

Received 17 May 2015; accepted 6 September 2015; published 9 September 2015

Copyright (C) 2015 by authors and Scientific Research Publishing Inc.

This work is licensed under the Creative Commons Attribution International License (CC BY). http://creativecommons.org/licenses/by/4.0/

(c) (i) Open Access

\begin{abstract}
Objectives: To study the reliability of a transcutaneous bilirubinometer (Bilicheck) to determine bilirubin levels in neonates consulting for jaundice in a Paediatric Emergency Department (ED), and to evaluate its usefulness as a screening method. Methods: Prospective observational study realized between June of 2005 and December of 2005 in neonates consulting at a paediatric emergency department for jaundice, in whom we realized both transcutaneous and total serum bilirubin measurements (TcB and TSB). We collected demographic variables, analytical variables (serum and transcutaneous bilirubin levels), length of stay in the ED, and need for treatment. Results: 66 children were included aged 2 to 31 days ( $81 \%$ of the sample were 2 to 7 days old). There was a close and statistically significant correlation between TcB and TSB $(r=0.81, p<0.001)$. The area under the ROC curve was of 0.90 , allowing detecting newborns with jaundice susceptible of treatment with TcB levels $\geq 13 \mathrm{mg} / \mathrm{dL}$ (sensitivity $92 \%$, specificity $63,5 \%$, a positive predictive value $39 \%$ and a negative predictive value $97 \%$ ). The number of venous punctures could be reduced in 50\%. The medium stay in the ED was of 2 hours when performing serum measurements. Conclusions: A linear correlation exists between TcB-TSB. TcB measurement cannot replace that of TsB, however it could be used as a screening method in an ED to determine which neonates need confirmation by TsB measurement. The use of transcutaneous bilirubinometer would reduce both the number of painful interventions in neonates and the medium length of stay in ED, consequently reducing iatrogenesis.
\end{abstract}

\section{Keywords}

Jaundice, Bilirubinometer, Screening, Transcutaneous Bilirubin, Seric Bilirubin

\footnotetext{
${ }^{*}$ Corresponding author.

How to cite this paper: Míguez, C., Salto, M.F. and Marañón, R. (2015) Measurement of Transcutaneous Bilirubin with Bilicheck as a Jaundice Screening Method in Neonates in Pediatric Emergency Departments. Open Journal of Pediatrics, 5, 240-247. http://dx.doi.org/10.4236/ojped.2015.53037
} 


\section{Introduction}

The yellowish coloration of skin and mucosa (jaundice) is a frequent diagnosis in neonates. This coloration can be observed when serum bilirubin levels are higher than $5 \mathrm{mg} / \mathrm{dL}$. Two thirds of all newborn infants are estimated to present a certain degree of jaundice during their first weeks of life. In a percentage of cases, bilirubin levels can exceed neurotoxic levels, with the subsequent risk of producing kernicterus [1]-[3].

Causes of jaundice in neonates are diverse, ranging from physiological jaundice which usually requires no treatment, to septic shock which requires hospitalization in intensive care units.

Evaluation of jaundice in a paediatric emergency department requires a precise medical history, physical examination and at times, laboratory tests.

Although intensity and localization of jaundice is commonly used as an indicator of bilirubin blood concentration, the correlation between visual estimation and actual bilirubin concentration is poor [4] [5].

In 2004, the American Academy of Paediatrics published clinical practice guidelines on management of neonatal hyperbilirubinemia, affirming that visual estimation of the degree of jaundice can lead to errors, therefore bilirubin levels should be determined by measurement of total serum bilirubin (TSB) or by transcutaneous bilirubin (TcB), and interpreted according to the age (in hours) of the newborn infant [1] [2].

Previous studies have demonstrated that TcB determinations represent good estimations of the TSB levels in term and late preterm neonates in their first days of life; however, they tend to underestimate TSB at high levels. Recent studies indicate that the TcB determination is more accurate than visual estimation methods [4] [6].

The principle objective of our study was to evaluate the reliability of a transcutaneous bilirubinometer (Bili Chek ${ }^{\circledR}$ ) and to determine its usefulness in an emergency department as a screening method in newborn infants susceptible to treatment. Secondary objectives were to find out whether the use of a transcutaneous bilirubinometer could reduce the number of venous punctures (without however affecting the percentage of admissions), as well as the average length of stay in the emergency department.

\section{Materials and Methods}

We performed a prospective observational study from June 2005 through December 2005 amongst neonates that consulted for jaundice in the Emergency Department. The study took place in the emergency service of the Hospital General Universitario Gregorio Marañón in Madrid, which usually attends to 60,000 children aged 2 days to 16 years.

The study meets the Helsinki Declaration standards and has been approved by our hospital's clinical investtigation and ethics committee.

The population we studied consisted of all children aged 2 to 30 days (both included) which consulted the emergency department for jaundice. We excluded preterm babies, infants aged more than 30 days, infants who had previously received phototherapy and neonates with clinical signs of dehydration or toxic aspect.

The variables collected in the study are shown in Table 1.

In all children included in the study, we measured both transcutaneous and serum bilirubin levels.

TSB measurement was made in our hospital's biochemistry laboratory by spectrophotometry.

TcB measurement was made with a transcutaneous bilirubinometer (Bilicheck, by Philips brand). The determination of TcB with the bilirubinometer was done by 5 skin measurements in the forehead, upper third of the thorax, areas free of hair or skin blemishes. The equipment then calculated the average of all 5 determinations in $\mathrm{mg} / \mathrm{dL}$.

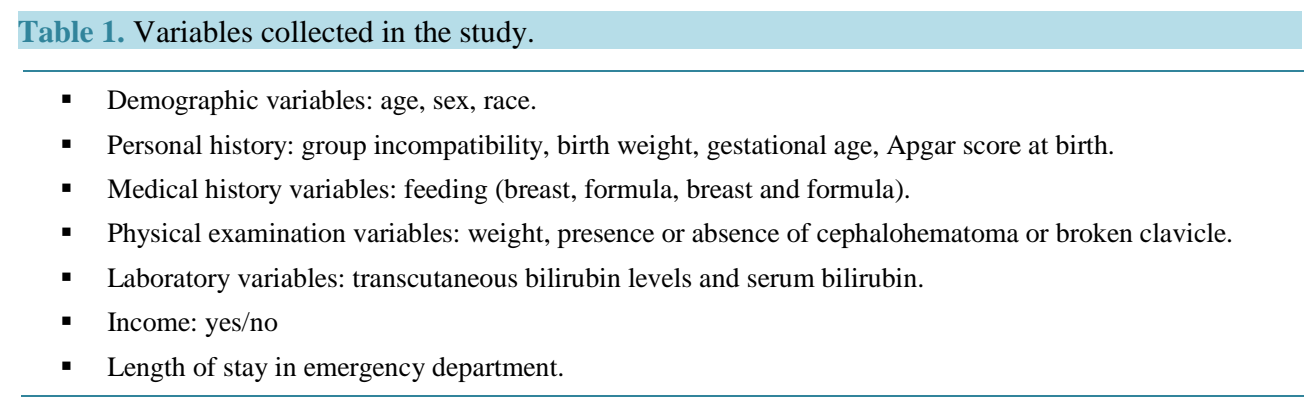


We considered infants older than 48 hours of life with bilirubin levels $\geq 18 \mathrm{mg} / \mathrm{dl}$ as being susceptible to treatment (we did not consider preterm, low weight at birth, nor group incompatibility).

For the administration of treatment the American Academic of Paediatrics guideline in management of hyperbilirubinemic in the newborn infant 35 or more weeks of gestation were followed.

The data was introduced in Access 2003 database and was then treated statistically with SPSS program version 14.

We performed a correlation, regression and variance analysis. With the use of a ROC curve we determined the sensitivity and specificity of transcutaneous bilirubin measurement, as well as the optimal cut-off point performing a checkout with serum bilirrubin in neonates with jaundice.

\section{Results}

66 children were included in the study (82\% of those who consulted for jaundice and met the inclusion criteria at the time of the study). The patients were aged 2 to 30 days of life. $81 \%$ of the samples were in their first week of life.

The demographic characteristics of the children in the study are shown in Table 2.

We compared TSB and TcB determinations in all patients, finding a statistically significant linear correlation between both measurements $\left(r=0.81, r^{2}=0.66, p<0.001\right)$, as shown in Figure 1 . The maximum and minimum difference found between the bilirubin numbers determined with both methods was of $-10 \mathrm{mg} / \mathrm{dL}$ and -0.4 $\mathrm{mg} / \mathrm{dL}$, suggesting that Bilicheck underestimates TSB levels.

Using variance analysis, we analysed whether demographic characteristics such as race, weight, presence or not of group incompatibility had an influence on the precision of TcB measurement. No influence was found.

With the use of a ROC curve we determined the sensitivity and specificity of transcutaneous bilirubin levels, as well as the optimal cut-off point for detecting jaundice requiring treatment. The area under the ROC curve was of 0.90 (95\% confidence interval [0.82 - 0.98]) (Figure 2).

In the coordinates table transcutaneous bilirubin levels (Table 3).

By analyzing the ROC curves optimal cutoff of the bilirrubin transcunateous with increased sensitivity, specificity and negative predictive value was detected in those neonates that they should perform serum bilirrubin to be eligible for treatment.

This optimal cutoff was $13 \mathrm{mg} / \mathrm{dl}$ transcuataneous bilirrubin corresponding to a $18 \mathrm{mg} / \mathrm{dl}$ serum bilirrubin (value close to the levels of phototherapy depending on the weight, age, gestational age, and days of life).

TcB levels of $13 \mathrm{mg} / \mathrm{dL}$ seems to be the optimal cut-off point for detecting need of treatment, with $92.9 \%$ sensitivity, $62.1 \%$ specificity, a positive predictive value of $39 \%$ and a negative predictive value of $97 \%$ (Table 4).

Half of our patients presented TcB levels $<13 \mathrm{mg} / \mathrm{dL}$, and were therefore not susceptible for treatment and did not need further confirmation with TSB determination. This leads to suppose that $50 \%$ of the neonates included

Table 2. The demographic characteristics of the children in the study.

\begin{tabular}{cc}
\hline Characteristics & Range or percentage (n) \\
\hline Gestational age & 37 - 41 sem \\
Birth weight & $2190-3920 \mathrm{gr}$ \\
Breastfeeding & $71 \%(47)$ \\
Breast and formula feeding & $19.6 \%(13)$ \\
formula feed & $9 \%(6)$ \\
Caucasian race & $74.2 \%(49)$ \\
Others races & $25.6 \%(17)$ \\
Blood group incompatibility & $13.6 \%(9)$ \\
Cephalohematoma & $3 \%(2)$ \\
Clavicle fracture & $3 \%(2)$ \\
Apgar score at birth $<7$ & $1.5 \%(1)$ \\
\hline
\end{tabular}


Table 3. Coordinates table transcutaneous bilirubin levels: optimal cut-off point for detecting need of treatment.

\begin{tabular}{cccc}
\hline Transcutaneous bilirubin levels $(\mathbf{m g} / \mathbf{d l})$ & Sensitivity & 1-Specificity \\
\hline 3.5 & 1.0 & 1.0 \\
5 & 1.0 & 0.98 \\
9.5 & 1.0 & 0.90 \\
10 & 1.0 & 0.86 \\
11 & 1.0 & 0.71 \\
12 & 1.0 & 0.5 \\
13 & $\mathbf{0 . 9 3}$ & 0.173 \\
14 & 0.85 & 0.077 \\
\hline
\end{tabular}

Table 4. Sensitivity, specificity, positive predictive value and negative predictive value of transcutaneous bilirubin level (13 $\mathrm{mg} / \mathrm{dl})$ for detection of jaundice phototerapy susceptible.

\begin{tabular}{ccccc}
\hline & Sensitivity & Specificity & Positive predictive value & Negative predictive value \\
\hline $\begin{array}{c}\text { Transcutaneous bilirrubin } \\
\text { level = 13 mg/dl }\end{array}$ & $93 \%$ & $62 \%$ & $39 \%$ & $97 \%$ \\
\hline
\end{tabular}

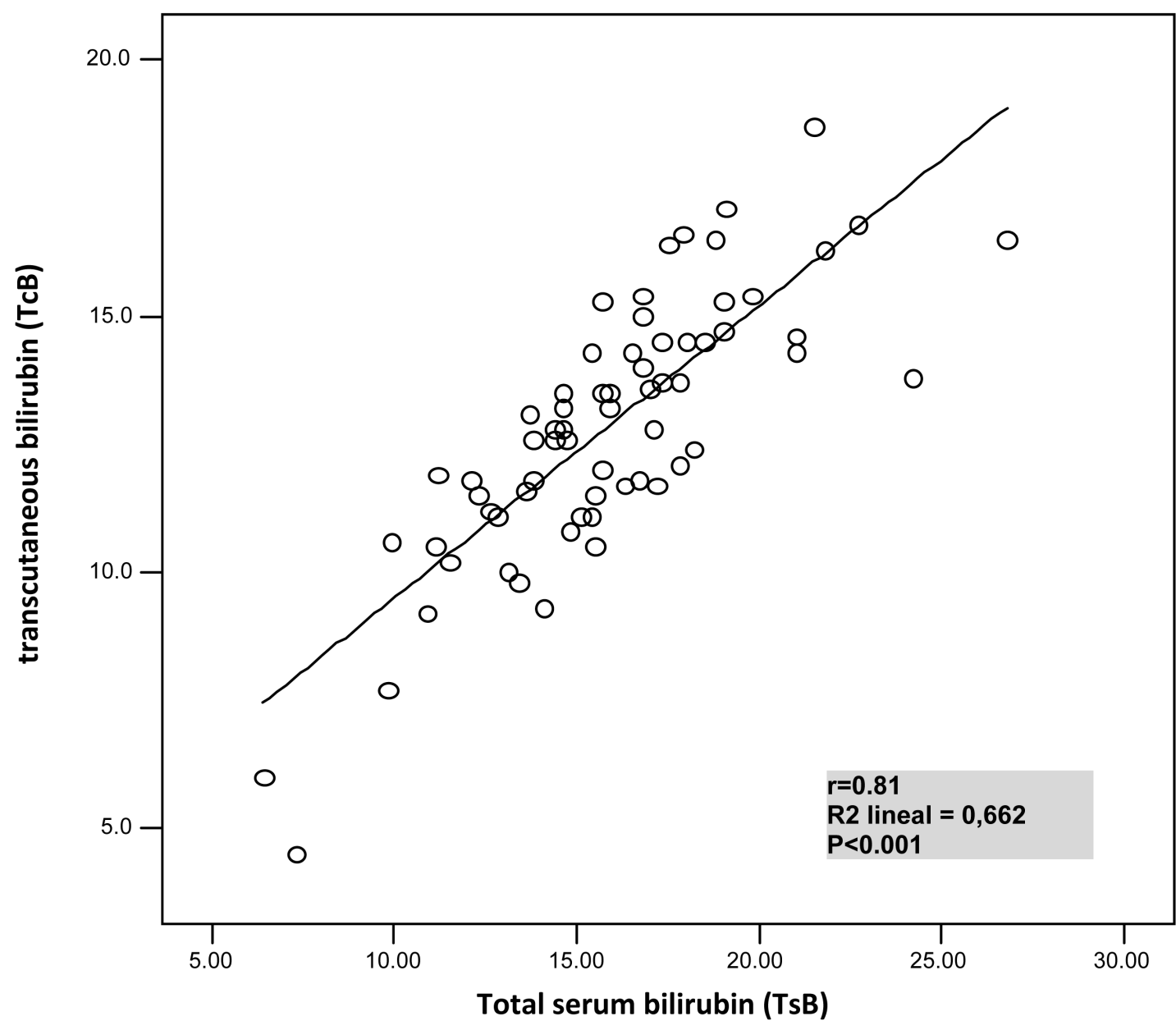

Figure 1. Comparison of total serum bilirrubin and transcutaenous bilirubin determinations $(r=0.81 ; p<0.001)$. 


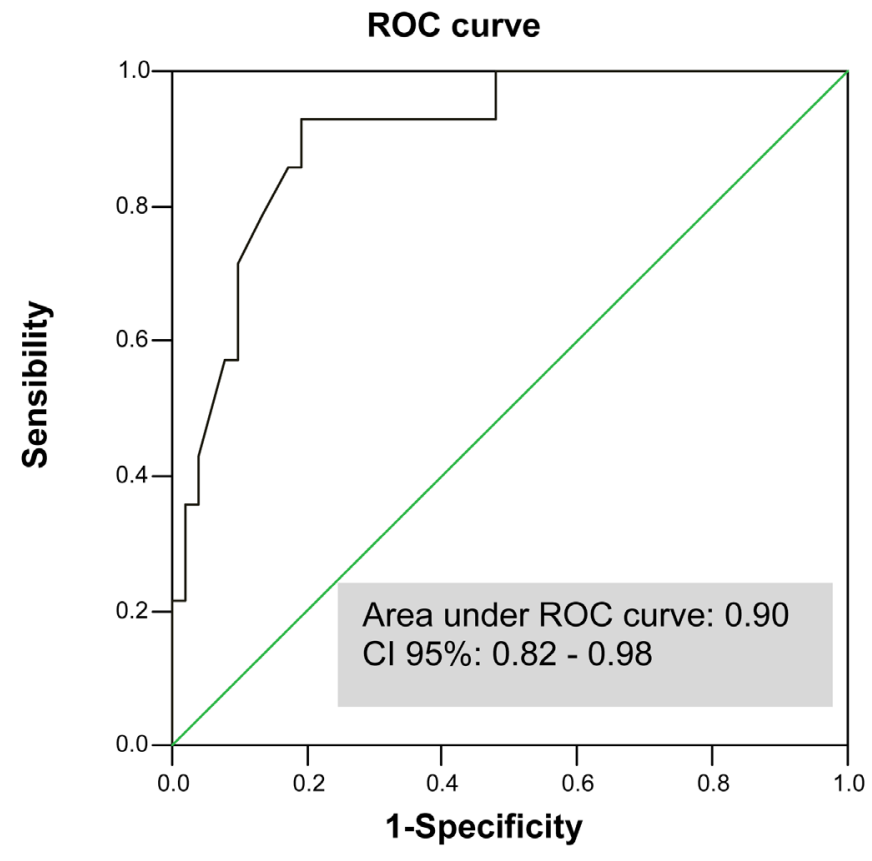

Figure 2. ROC curve for transcutaneous bilirubin levels.

in this study didn't actually need a venous puncture to determine serum bilirubin level.

In 61 of all 66 patients, we noted the time of entrance and time of exit of the Emergency Department, observing a mean time of stay of 2 hours when performing venous puncture for the determination of TSB (minimum 50 minutes, maximum 4 hours).

\section{Discussion}

The evaluation of the efficacy of non invasive methods for the determination of hyperbilirubinemia in the jaundice neonate is of great importance in daily clinical practice.

Non-invasive bilirubin measurement methods include visual estimation of hyperbilirubinemia and TcB determination with a bilirubinometer.

The visual estimation of hyperbilirubinemia has shown to correlate to TSB in various studies [4] [6], however it has limitations such as the difficulty in indentifying jaundice in dark-skinned babies [5]. Recent studies have shown that TcB determination has a better correlation than visual estimation methods [4] [6].

As in previous studies, we have found a statistically significant linear correlation between TSB and TcB determination with Bilicheck. However, this correlation decreases as serum bilirubin levels increase, which seems to affect Bilicheck's precision.

In our study, a sub estimation in bilirubin levels is observed when using Bilicheck, as in previous studies [7][9]. The cut-off point obtained was of $13 \mathrm{mg} / \mathrm{dL}$. Higher levels obtained with our bilirubinometer determined a higher probability of having TSB levels susceptible to treatment. In these cases, a serum bilirubin determination should be performed to confirm TcB results.

In the last few years, different systems have been designed to measure bilirubin levels transcutaneous. Those that have been the most studied (Minolta/Hill-Room Air Shields Transcutaneous Jaundice Meter-103 and Bilicheck) have shown a good correlation with serum levels of bilirubin and have proven to be useful screening methods [10]-[14].

The impossibility to measure bilirubin fractions (direct, indirect), and the fact that it cannot be used if the patient has previously received treatment with phototherapy are some of the limitations linked to the use of bilirubinometers.

According to the AAP guidelines, serum bilirubin should be measured in case of high TcB levels, toxic or septic aspect, infants older than 3 weeks old, or if a direct bilirubin determination is needed [1]-[3].

The bilirubinometer used in our study (Bilicheck) measures TcB using full-spectrum light (380 to $760 \mathrm{~nm}$ ) re- 
flected by the skin. The use of a multiple-spectrum wavelength allows to distinguish different optical densities due to bilirubin and other possible confusion factors such as haemoglobin, melanin, skin depth. This method allows thus to quantify tissue and capillary bilirubin omitting confusion factors. This measurement is therefore theoretically independent of ethnic origin, age and weight of the newborn [10]-[13].

High resolution liquid chromatography is the reference technique for measuring TSB. Bilicheck uses it as a standard reference. This technique is scarcely used in laboratories due to its technical difficulties and complicated use on a daily basis. The use of different laboratory techniques might explain the different correlations between TcB and TSB in different studies. In our hospital for example, our laboratory uses direct spectrophotometry for the measurement of TSB, with which we have correlated Bilicheck findings in our study.

In our sample, as well as in a study published by Maria Das Graças da Cunha Leite et al., the correlation between bilirubin levels measured with Bilicheck and serum bilirubin was not affected by race, age or weight at birth [15] [16].

In the case of premature babies, various studies have demonstrated the efficacy of transcutaneous bilirubinometer in this subgroup [17] [18]. However a few studies refer that race and skin colour might affect the measurement of TcB in this subgroup [19]-[22].

The use of a transcutaneous bilirubinometer as a screening method decreases the number of venous and capillary punctures in neonates with jaundice [7] [16].

In our study if the bilirubinometer had been used to determine the need for a serum bilirubin determination in infants with TcB $13 \mathrm{mg} / \mathrm{dL}$ or higher, the number of blood tests would have been reduced in $50 \%$ and only one patient actually needing phototherapy would have been lost. In a study published by Samanta et al. [7] where TcB cut-off point was $14 \mathrm{mg} / \mathrm{dL}$, the number of venous punctures was reduced in $55 \%$.

Reducing venous and capillary punctures in these children would reduce pain and iatrogenesis and would increase the personnel and parents' satisfaction.

In our study, we recorded the time of admission and discharge for each child, to evaluate the average time of stay needed for the measurement of TSB in infants with jaundice. The average stay was of 2 hours (minimum 50 minutes, maximum 4 hours). This time would have been reduced if the need for a serum determination had been checked beforehand with Bilicheck.

In some studies, the reduction in the length of stay in the emergency department has proven to be one of the utilities of the transcutaneous bilirubinometer in the emergency ward [23] [24].

This reduction in the length of stay allows a better flow of patients amongst an emergency department, and reduces the risk of transmission of infectious diseases.

\section{Limitations}

Although Bilicheck was used in all cases to determine TcB, measurements were obtained by different operators, which could have led to errors.

\section{Conclusions}

Although Bilicheck cannot replace the measurement of serum bilirubin as a reference test, the correlation found between these two methods shows that it could be an interesting tool to discriminate neonates in need of a blood test to evaluate jaundice.

The use of TcB as a screening method in neonates that consult in an emergency department would reduce the use of invasive methods, iatrogenesis, and length of stay in the department and would allow starting treatments more promptly. In this case, TSB measurement should be performed if TcB is higher that $13 \mathrm{mg} / \mathrm{dL}$, in children who have been treated previously with phototherapy or if a toxic aspect is detected.

\section{Acknowledgements}

The authors would like to thank Dr. Julie Cayrol. M. D. Pediatric Emergency Department. Hospital General Universitario Gregorio Marañón. Madrid who translation of the text into English.

\section{Declaration}

No sponsorship or conflicts of interest were declared by the authors. 


\section{Conflicts of Interest}

The authors declare that they have no conflicts of interest.

\section{References}

[1] American Academy of Pediatrics, Subcommittee on Hyperbilirubinemia (2004) Management of Hyperbilirubinemia in the Newborn Infant 35 or More Weeks of Gestation [Published Correction Appears in Pediatrics. 2004, 114, 1138]. Pediatrics, 114, 297-316. http://dx.doi.org/10.1542/peds.114.1.297

[2] Canadian Paediatric Society, Fetus and Newborn Committee (2007) Guidelines for Detection, Management and Prevention of Hyperbilirubinemia in Term and Late Preterm Newborn Infants (35 or More Weeks' Gestation). Paediatr Child Health, 12, 401-407.

[3] Maisels, M.J., Bhutani, V.K., Bogen, D., et al. (2009) Hyperbilirubinemia in the Newborn Infant 9 or $=35$ Weeks' Gestation: An Update with Clarifications. Pediatrics, 124, 1193-1198. http://dx.doi.org/10.1542/peds.2009-0329

[4] Keren, R., Tremont, K., Luan, X. and Cnaan, A. (2009) Visual Assessment of Jaundice in Term and Late Preterm Infants. Archives of Disease in Childhood_Fetal and Neonatal Edition, 94, F317-F322. http://dx.doi.org/10.1136/adc.2008.150714

[5] Kaplan, M., Shchors, I., Algur, N., et al. (2008) Visual Screening versus Transcutaneous Bilirubinometry for Predischarge Jaundice Assessment. Acta Paediatrica, 97, 759-763. http://dx.doi.org/10.1111/j.1651-2227.2008.00807.x

[6] Moyer, V.A., Ahn, C. and Sneed, S. (2000) Accuracy of Clinical Judgment in Neonatal Jaundice. Archives of Pediatrics and Adolescent Medicine, 154, 391-394.

[7] Samanta, S., Tan, M., Kissack, C., et al. (2004) The Value of Bilicheck as Screening Tool for Neonatal Jaundice in Term and Near-Term Babies. Acta Paediatrica, 93, 1486-1490. http://dx.doi.org/10.1111/j.1651-2227.2004.tb02634.x

[8] Nanjundaswamy, S., Petrova, A., Mehta, R., Bernstein, W. and Hegyi, T. (2004) The Accuracy of Transcutaneous Bilirubin Measurements in Neonates: A Correlation Study. Biology of the Neonate, 85, 21-25. http://dx.doi.org/10.1159/000074953

[9] Wong, C.M., van Dijk, P.J.E. and Laing, I.A. (2002) A Comparison of Transcutaneous Bilirubinometers: SpectRx BiliCheck versus Minolta AirShields. Archives of Disease in Childhood_Fetal and Neonatal Edition, 87, 137-140. http://dx.doi.org/10.1136/fn.87.2.F137

[10] Bhutani, V.K., Gourley, G.R., Adler, S., Kreamer, B., Dalin, C. and Johnson, L.H. (2000) Noninvasive Measurement of Total Serum Bilirubin in a Multiracial Predischarge Newborn Population to Assess the Risk of Severe Hyperbilirubinemia. Pediatrics, 106, E17. http://dx.doi.org/10.1542/peds.106.2.e17

[11] Maisels, M.J. (2006) Transcutaneous Bilirubinometry. Neoreviews, 7, e217-e225. http://dx.doi.org/10.1542/neo.7-5-e217

[12] Samanta, S., Tan, M., Kissack, C., Nayak, S., et al. (2004) The Value of Bilicheck as a Screening Tool for Neonatal Jaundice in Term an Near-Term Babies. Acta Paediatrica, 93, 1486-1490. http://dx.doi.org/10.1111/j.1651-2227.2004.tb02634.x

[13] Rubaltelli, F., Gourley, G., Loskamp, N., et al. (2001) Transcutaneous Bilirrbin Measurement a Multicenter Evaluation of a New Device. Pediatrics, 107, 1264-1271. http://dx.doi.org/10.1542/peds.107.6.1264

[14] Thayyil, S. and Marriott, L. (2005) Can Transcutaneous Bilirubinometry Reduce the Need for Serum Bilirubin Estimations in Term and near Term Infants? Archives of Disease in Childhood, 90, 1311-1312. http://dx.doi.org/10.1136/adc.2004.070292

[15] Bertini, G. and Rubaltelli, F. (2002) Non-Invasive Bilirubinometry in Neonatal Jaundice. Seminars in Neonatology, 7, 129-133. http://dx.doi.org/10.1053/siny.2002.0100

[16] da Cunha Leite, M. das G., de Araújo Granato, V., Facchini, F.P. and Marba, S.T.M. (2007) Comparison of Transcutaneous and Plasma Bilirubin Measurement. Jornal de Pediatria, 83, 283-286. http://dx.doi.org/10.2223/JPED.1619

[17] Fouzas, S., Karatza, A.A., Skylogianni, E., Mantagou, L. and Varvarigou, A. (2010) Transcutaneous Bilirubin Levels in Late Preterm Neonates. Journal of Pediatrics, 157, 762-766. http://dx.doi.org/10.1016/j.jpeds.2010.04.076

[18] Zecca, E., Barone, G., De Luca, D., Marra, R., Tiberi, E. and Romagnoli, C. (2009) Skin Bilirubin Measurement during Phototherapy in Preterm and Term Newborn Infants. Early Human Development, 85, 537-540. http://dx.doi.org/10.1016/j.earlhumdev.2009.05.010

[19] Yu, Z.B., Dong, X.Y., Han, S.P., Chen, Y.L., Qiu, Y.F., Sha, L., et al. (2011) Transcutaneous Bilirubin Nomogram for Predicting Neonatal Hyperbilirubinemia in Healthy Term and Late-Preterm Chinese Infants. European Journal of Pediatrics, 170, 185-191. http://dx.doi.org/10.1007/s00431-010-1281-9

[20] Mishra, S., Chawla, D., Agarwal, R., Deorari, A.K. and Paul, V.K. (2010) Transcutaneous Bilirubin Levels in Healthy 
Term and Late Preterm Indian Neonates. Indian Journal of Pediatrics, 77, 45-50. http://dx.doi.org/10.1007/s12098-010-0007-3

[21] Wainer, S., Rabi, Y., Parmar, S.M., Allegro, D. and Lyon, M. (2009) Impact of Skin Tone on the Performance of a Transcutaneous Jaundice Meter. Acta Paediatrica, 98, 1909-1915. http://dx.doi.org/10.1111/j.1651-2227.2009.01497.x

[22] De Luca, D., Jackson, G.L., Tridente, A., Carnielli, V.P. and Engle, W.D. (2009) Transcutaneous Bilirubin Nomograms: A Systematic Review of Population Differences and Analysis of Bilirubin Kinetics. Archives of Pediatrics \& Adolescent Medicine, 163, 1054-1059. http://dx.doi.org/10.1001/archpediatrics.2009.187

[23] Lam, T.S., Tsui, K.L. and Kam, C.W. (2008) Evaluation of a Point of Care Transcutaneous Bilirubinometer in Chinese Neonates at an Accident and Emergency Department. Hong Kong Medical Journal, 14, 356-360.

[24] Yamamoto, L.G., Killeen, J. and French, G.M. (2012) Transcutaneous Bilirubin Measurement Methods in Neonates and Its Utility for Emergency Department Use. Pediatric Emergency Care, 28, 380-387. http://dx.doi.org/10.1097/PEC.0b013e31824dcb43 